\title{
RADIO GALAXIES AND RELATIVISTIC BEAMING
}

\author{
D. J. Saikia \\ University of Manchester \\ Cheshire, England
}

There have been recent suggestions and suspicions that the earlier unified scheme, which largely involved quasars, could be enlarged upon to include galaxies as well. In this scheme, the extended radio galaxies are believed to be c'ose to the plane of the sky while the lobe and core-dominated quasars are seen at progressively smaller angles to the line-of-sight. In this paper, we study the correlations of the degree of core prominence with optical properties as well as radio structures on VLBI and larger scales for the both radio galaxies and quasars, in order to investigate whether they are consistent with the enlarged unified sche..e. From earlier statistical studies of quasar samples, the degree of core prominence has been shown to be a reasonable statistical indicator of source orientation (cf., Saikia 1985 and references therein). Saikia (1984) also showed that jets in quasars are more easily detected in the ones with more prominent cores, consistent with the unified scheme. In the present paper, we present the results of our observations of 30 lowluminosity radio galaxies and investigate whether such a result holds for galaxies as well.

\section{REFERENCES}

Saikia, D. J., 1984, M. N. R. A. S., 208, 231.

Saikia, D. J., 1985, Extragalactic Finergetic Sources, ed. V. K. Kapahi (Bangalore: Indian Academy of Sciences). 